\title{
Research Article \\ Periodic Problems of Difference Equations and Ergodic Theory
}

\author{
B. A. Biletskyi, ${ }^{1}$ A. A. Boichuk, ${ }^{2}$ and A. A. Pokutnyii ${ }^{2}$ \\ ${ }^{1}$ Department of Inductive Modeling and Control Glushkov, Institute of Cybernetics NAS Ukraine, \\ No. 40 Glushkov Avenue, Kyiv 03680, Ukraine \\ ${ }^{2}$ Laboratory of boundary value problems of differential equations theory, \\ Institute of Mathematics of NAS of Ukraine, No. 3 Tereshenkivska Street, Kyiv 01601, Ukraine
}

Correspondence should be addressed to A. A. Boichuk, boichuk@imath.kiev.ua

Received 1 February 2011; Accepted 11 May 2011

Academic Editor: Miroslava Růžičková

Copyright (C 2011 B. A. Biletskyi et al. This is an open access article distributed under the Creative Commons Attribution License, which permits unrestricted use, distribution, and reproduction in any medium, provided the original work is properly cited.

The necessary and sufficient conditions for solvability of the family of difference equations with periodic boundary condition were obtained using the notion of relative spectrum of linear bounded operator in the Banach space and the ergodic theorem. It is shown that when the condition of existence is satisfied, then such periodic solutions are built using the formula for the generalized inverse operator to the linear limited one.

\section{The Problem and The Main Statement}

The problem of existence of periodic solutions for classes of equations is well known. Though it is hard to mention all the contributors in a single paper, we would like to mark out welldeveloped Floke theory [1], which is used in analysis of linear differential equation systems by the means of monodromy matrix. Operator analogy of such theory in noncritical case (when there is single solution) for differential equations in Banach space was developed by Daletskyi and Krein [2].

This paper is dedicated to obtaining analogous conditions for a family of difference equations in Banach space and to building representations of corresponding solutions. The proposed approach allows obtaining solutions for both critical and noncritical cases. Note that this problem can be approached using well-developed pseudoinverse techniques in theory of boundary value problems [3]. In this paper we firstly build a new representation of the pseudoinverse operator based on results of ergodic theory, and then we provide the necessary and sufficient conditions that guarantee the existence of the corresponding solutions. 
Let B-complex Banach space with norm $\|\cdot\|$ and zero-element $\overline{0} ; \mathcal{L}(\mathbf{B})$-Banach space of bounded linear operators from $\mathbf{B}$ to $\mathbf{B}$. In this paper we consider existence of periodic solutions of the equation

$$
x_{n+1}=\lambda A_{n+1} x_{n}+h_{n+1}, \quad n \geqslant 0,
$$

with periodicity condition

$$
x_{0}=x_{m}
$$

where $A_{n} \in \mathcal{L}(\mathbf{B}), A_{n+m}=A_{n}$, for all $n \geqslant 0, \lambda$ is a complex parameter, and $\left\{h_{n}\right\}_{n=0}^{\infty}$ is a sequence in $\mathbf{B}$. The solution of the corresponding homogeneous equation to (1.1) has the following form [4]:

$$
x_{m}(\lambda)=\Phi(m, n, \lambda) x_{n}(\lambda), \quad m \geqslant n,
$$

where

$$
\Phi(m, n, \lambda)=\lambda^{m-n} A_{m+1} A_{m} \cdots A_{n+1}, \quad m>n
$$

is evolution operator for problem $(1.1) ; \Phi(m, m, \lambda)=E$, where $E$ is identity operator. Let us remark that $U(m, \lambda)=\Phi(m, 0, \lambda), U(0, \lambda)=E$ and $U(k+n, \lambda)=U(k, \lambda) U(n, \lambda)$. Operator $U(m, \lambda)$ is traditionally called monodromy operator.

We can represent [4] the solution (1.1) with arbitrary initial condition $x(0, \lambda)=x_{0}, x_{0} \in$ $\mathbf{B}$ in the form

$$
x_{k}(\lambda)=\Phi(k, 0, \lambda) x_{0}+g(k, \lambda)
$$

where

$$
g(k, \lambda)=\sum_{i=0}^{k} \Phi(k, i, \lambda) h_{i}
$$

If we substitute this representation in boundary condition (1.2), we obtain operator equation

$$
x_{0}(\lambda)-x_{m}(\lambda)=x_{0}-\Phi(m, 0, \lambda) x_{0}-g(m, \lambda)=\overline{0} .
$$

According to notations, we get operator equation

$$
(E-U(m, \lambda)) x_{0}=g(m, \lambda) .
$$

Boundary value problem (1.1), (1.2) has periodic solution if and only if operator equation (1.8) is solvable. 
Following the paper [5], point $\lambda$ is called right stable point if monodromy operator satisfies inequality $\left\{\left\|U^{n}(m, \lambda)\right\| \leqslant c, n \geqslant 0\right\}$.

Denote $\rho_{N S}(E-U(m, \lambda))=\{\lambda \in \mathbb{C}: R(E-U(m, \lambda))=\overline{R(E-U(m, \lambda))}\}$ (this set coincides with the set of all $\lambda \in \mathbb{C}$ such that operator $E-U(m, \lambda)$ is normally solvable). It follows easily that resolvent set $\rho(E-U(m, \lambda))$ of the operator $E-U(m, \lambda)$ lies in $\rho_{N S}(E-$ $U(m, \lambda))$.

In the sequel we assume that $\mathbf{B}$ is reflexive for simplicity [6].

The main result of this paper is contained in Theorem 1.1.

Theorem 1.1. Let $\lambda \in \rho_{N S}(E-U(m, \lambda))$ be right stability point for (1.1). Then

(a) boundary value problem (1.1), (1.2) has solutions if and only if sequence $\left\{h_{n}\right\}_{n \in \mathbb{Z}_{+}}, h_{n} \in \mathbf{B}$ satisfies condition

$$
\lim _{n \rightarrow \infty} \frac{\sum_{k=1}^{n} \sum_{i=0}^{m} U^{k}(m, \lambda) \Phi(m, i, \lambda) h_{i}}{n}=0
$$

(b) under condition (1.9), solutions of boundary value problem (1.1), (1.2) have the following form:

$$
x_{n}=U(n, \lambda) \lim _{k \rightarrow \infty} \frac{\sum_{m=1}^{k} U^{m}(k, \lambda)}{k} c+U(n, \lambda) G(n, \lambda)\left[h_{n}\right]
$$

where $c$ is an arbitrary element of Banach space B, $G(n, \lambda)$-generalized Green operator of boundary value (1.1), (1.2), which is defined by equality

$$
\begin{aligned}
G(n, \lambda)\left[h_{n}\right]= & \sum_{k=0}^{\infty}(1-\mu)^{k}\left\{\sum_{l=0}^{\infty} \mu^{-l-1}\left(U(m, \lambda)-U_{0}(\lambda)\right)^{l}\right\}^{k+1} \sum_{i=0}^{m} \Phi(m, i, \lambda) h_{i} \\
& -U_{0}(\lambda) \sum_{i=0}^{m} \Phi(m, i, \lambda) h_{i}+\sum_{i=0}^{n} \Phi(n, i, \lambda) h_{i} .
\end{aligned}
$$

\section{Auxiliary Result}

Let us formulate and prove a number of auxiliary lemmas, which entail the theorem.

Lemma 2.1. If $\lambda \in \rho_{N S}(E-U(m, \lambda))$, then boundary value problem (1.1), (1.2) is solvable if and only if sequence $h_{n}$ satisfies the condition

$$
\lim _{n \rightarrow \infty} \frac{\sum_{k=1}^{n} \sum_{i=0}^{m} U^{k}(m, \lambda) \Phi(m, i, \lambda) h_{i}}{n}=0 .
$$


Proof. From the assumption above it follows that the conditions of statistical ergodic theorem hold [6]. Then

$$
R(E-U(m, \lambda))=\left\{x \in \mathbf{B}: \lim _{n \rightarrow \infty} U_{n}(m, \lambda) x=\overline{0}, U_{n}(m, \lambda)=\frac{\sum_{k=1}^{n} U^{k}(m, \lambda)}{n}\right\} .
$$

It follows from the equation above that element $g(m, \lambda)$ lies in value set of the operator $E-$ $U(m, \lambda)$ if and only if

$$
\lim _{n \rightarrow \infty} \frac{\sum_{k=1}^{n} U^{k}(m, \lambda)}{n} \sum_{i=0}^{m} \Phi(m, i, \lambda) h_{i}=0,
$$

which proves the lemma.

Consider the following consequences of the assumptions above for further reasoning. Suppose that $\lambda \in \rho_{N S}(E-U(m, \lambda))$ and $\lambda$ is right stable point of the monodromy operator, such that $\lambda$ define eigenspace $N(E-U(m, \lambda)$ ), which coincides with the values set of operator $U_{0}(\lambda) x=\lim _{n \rightarrow \infty} U_{n}(m, \lambda) x$. This operator satisfies the following conditions [6]:

(i) $U_{0}(\lambda)=U_{0}^{2}(\lambda)$

(ii) $U_{0}(\lambda)=U(m, \lambda) U_{0}(\lambda)$ (iii) $U_{0}(\lambda)=U_{0}(\lambda) U(m, \lambda)$

Lemma 2.2. Operator $E-U(m, \lambda)+U_{0}(\lambda): \mathbf{B} \rightarrow \mathbf{B}$ has bounded inverse of the form

$$
\left(E-U(m, \lambda)+U_{0}(\lambda)\right)^{-1}=\sum_{k=0}^{\infty}(\mu-1)^{k}\left\{\sum_{l=0}^{\infty} \mu^{-l-1}\left(U(m, \lambda)-U_{0}(\lambda)\right)^{l}\right\}^{k+1}
$$

for all $\mu>1:|1-\mu|<1 /\left\|R_{\mu}\right\|$.

Proof. Let us show that $\operatorname{Ker}\left(I-U(m, \lambda)+U_{0}(\lambda)\right)=\overline{0}$. Indeed, if $x \in \operatorname{Ker}\left(I-U(m, \lambda)+U_{0}(\lambda)\right)$, then

$$
\left(I-U(m, \lambda)+U_{0}(\lambda)\right) x=\overline{0}
$$

Since $(I-U(m, \lambda)) x \in \operatorname{Im}(I-U(m, \lambda))$ and $U_{0}(\lambda) x \in \operatorname{Ker}(I-U(m, \lambda))[6]$, subspaces $\operatorname{Im}(I-$ $U(m, \lambda))$ and $\operatorname{Ker}(I-U(m, \lambda))$ intersect only at zero point, and condition (2.6) is satisfied if and only if $(I-U(m, \lambda)) x=\overline{0}$ and $U_{0}(\lambda) x=\overline{0}$. This is possible if and only if $x=\overline{0}$. Let us show that $\operatorname{Im}\left(I-U(m, \lambda)+U_{0}(\lambda)\right)=\mathbf{B}$. Note $[6] \mathbf{B}=\operatorname{Ker}(I-U(m, \lambda)) \oplus \operatorname{Im}(I-U(m, \lambda))=\operatorname{Im}\left(U_{0}(\lambda)\right) \oplus$ $\operatorname{Im}(I-U(m, \lambda))$. It follows from the last decomposition that any element $x \in \mathbf{B}$ has the form 
$(I-U(m, \lambda)) y+U_{0}(\lambda) z$, where $y, z \in \mathbf{B}$, which proves that $\operatorname{Im}\left(I-U(m, \lambda)+U_{0}(\lambda)\right)=\mathbf{B}$. Hence according to the Banach theorem [6] original operator has inverse since it bijectively maps B to itself. Therefore point $\mu=1$ is regular [6] for the operator $\mu I-U(m, \lambda)+U_{0}(\lambda)$. Since powers of the operator $U(m, \lambda)$ are uniformly bounded and spectral radius $r_{U(m, \lambda)} \leqslant 1$ $\left(\sqrt[n]{\left\|U(m, \lambda)^{n}\right\|} \leqslant \sqrt[n]{c}\right.$, then $\left.r_{U(m, \lambda)}=\lim _{n \rightarrow \infty} \sqrt[n]{\left\|U(m, \lambda)^{n}\right\|} \leqslant \lim _{n \rightarrow \infty} \sqrt[n]{c}=1\right)$. It is well known [6] that resolvent set of a bounded operator is open. Number $\mu=1 \in \rho\left(U(m, \lambda)-U_{0}(\lambda)\right)$; thus there exist a neighborhood of $\mu$ such that each point from the neighborhood belongs to resolvent set. For any point $\mu>r_{\left(U(m, \lambda)-U_{0}(\lambda)\right)}$ that belongs to the neighborhood there exists a resolvent [6], which has the form of converging in the norm series

$$
R_{\mu}:=R_{\mu}\left(U(m, \lambda)-U_{0}(\lambda)\right)=\sum_{l=0}^{\infty} \mu^{-l-1}\left(U(m, \lambda)-U_{0}(\lambda)\right)^{l}
$$

Using the analyticity of the resolvent and well-known identity for points $\mu>1$ such that $|1-\mu|<1 /\left(\left\|R_{\mu}\left(U(m, \lambda)-U_{0}(\lambda)\right)\right\|\right)$, we obtain

$$
R_{1}=\sum_{k=0}^{\infty}(\mu-1)^{k} R_{\mu}^{k+1}
$$

Finally, by substituting the series in the equation above, we get (2.5), which proves the lemma.

Let us introduce some notation first before proving next statement.

Definition 2.3. Operator $L^{-} \in \mathcal{L}(\mathbf{B})$ is called generalized inverse for operator $L \in \mathcal{L}(\mathbf{B})$ [3] if the following conditions hold:

$$
\text { (1) } \quad L L^{-} L=L, \quad \text { (2) } \quad L^{-} L L^{-}=L^{-} \text {. }
$$

Lemma 2.4. Operator $E-U(m, \lambda)$ is generalized inverse and

$$
(E-U(m, \lambda))^{-}=\left(E-U(m, \lambda)+U_{0}(\lambda)\right)^{-1}-U_{0}(\lambda),
$$

or in the form of converging operator series

$$
(E-U(m, \lambda))^{-}=\sum_{k=0}^{\infty}(\mu-1)^{k}\left\{\sum_{l=0}^{\infty} \mu^{-l-1}\left(U(m, \lambda)-U_{0}(\lambda)\right)^{l}\right\}^{k+1}-U_{0}(\lambda),
$$

for all $\mu>1:|1-\mu|<1 /\left\|R_{\mu}\right\|$. 
Proof. It suffices to check conditions (1) and (2) of the Definition 2.3. We use both representations (2.10), (2.11) and the expression (2.4) for operator $U_{0}(\lambda)$. Consider the following product:

$$
\begin{aligned}
(I-U & (m, \lambda))\left(\left(I-U(m, \lambda)+U_{0}(\lambda)\right)^{-1}-U_{0}(\lambda)\right)(I-U(\lambda)) \\
= & \left(\left(I-U(m, \lambda)+U_{0}(\lambda)\right)-U_{0}(\lambda)\right) \times\left(\left(I-U(m, \lambda)+U_{0}(\lambda)\right)^{-1}-U_{0}(\lambda)\right)(I-U(m, \lambda)) \\
= & \left(I-U_{0}(\lambda)\left(I-U(m, \lambda)+U_{0}(\lambda)\right)^{-1}-\left(I-U(m, \lambda)+U_{0}(\lambda)\right) U_{0}(\lambda)+U_{0}(\lambda)^{2}\right) \\
& \times(I-U(m, \lambda)) \\
= & \left(I-U_{0}(\lambda)\left(I-U(m, \lambda)+U_{0}(\lambda)\right)^{-1}\right) \times(I-U(m, \lambda)) \\
= & \left(I-U_{0}(\lambda)\left(I-U(m, \lambda)+U_{0}(\lambda)\right)^{-1}\right)\left(\left(I-U(m, \lambda)+U_{0}(\lambda)\right)-U_{0}(\lambda)\right) \\
= & I-U(m, \lambda)+U_{0}(\lambda)-U_{0}(\lambda)-U_{0}(\lambda)+U_{0}(\lambda)\left(I-U(m, \lambda)+U_{0}(\lambda)\right)^{-1} U_{0}(\lambda) \\
= & I-U(m, \lambda)-U_{0}(\lambda)+U_{0}(\lambda)\left(I-U(m, \lambda)+U_{0}(\lambda)\right)^{-1} U_{0}(\lambda) .
\end{aligned}
$$

Note that $U_{0}(\lambda)\left(U(m, \lambda)-U_{0}(\lambda)\right)^{l}=0$ for any $l \in \mathbb{N}$ (this directly follows from (2.4) using formula of binominal coefficient). Now, prove that

$$
\begin{aligned}
U_{0}(\lambda)\left(I-U(m, \lambda)+U_{0}(\lambda)\right)^{-1} U_{0}(\lambda) & =U_{0}(\lambda)\left(I-U(m, \lambda)+U_{0}(\lambda)\right)^{-1} \\
& =\left(I-U(m, \lambda)+U_{0}(\lambda)\right)^{-1} U_{0}(\lambda) \\
& =U_{0}(\lambda) .
\end{aligned}
$$

Indeed

$$
\begin{aligned}
U_{0}(\lambda)\left(I-U(m, \lambda)+U_{0}(\lambda)\right)^{-1} U_{0}(\lambda) & =\sum_{k=0}^{\infty}(\mu-1)^{k} U_{0}(\lambda)\left\{\sum_{l=0}^{\infty} \mu^{-l-1}\left(U(m, \lambda)-U_{0}(\lambda)\right)^{l}\right\}^{k+1} U_{0}(\lambda) \\
& =\sum_{k=0}^{\infty}\left(\left(\mu^{-1}\right)^{k+1}(\mu-1)^{k} U_{0}(\lambda)+(\mu-1)^{k} U_{0}(\lambda)\right. \\
& \left.\times\left\{\sum_{l=1}^{\infty} \mu^{-l-1}\left(U(m, \lambda)-U_{0}(\lambda)\right)^{l}\right\}^{k+1}\right) U_{0}(\lambda) \\
& =\sum_{k=0}^{+\infty} \mu^{-k-1}(\mu-1)^{k} U_{0}(\lambda) \\
& =\frac{1}{\mu} \sum_{k=0}^{+\infty}\left(\frac{\mu-1}{\mu}\right)^{k} U_{0}(\lambda) \\
& =\frac{1}{\mu} \frac{1}{1-(\mu-1) / \mu} U_{0}(\lambda) \\
& =U_{0}(\lambda) .
\end{aligned}
$$


Thus

$$
I-U(m, \lambda)-U_{0}(\lambda)+U_{0}(\lambda)\left(I-U(m, \lambda)+U_{0}(\lambda)\right)^{-1} U_{0}(\lambda)=I-U(m, \lambda) .
$$

We have that the operator $I-U(m, \lambda)$ satisfies condition (1) of the Definition 2.3. Let us check condition (2)

$$
\begin{aligned}
((I- & \left.\left.U(m, \lambda)+U_{0}(\lambda)\right)^{-1}-U_{0}(\lambda)\right)(I-U(m, \lambda))\left(\left(I-U(m, \lambda)+U_{0}(\lambda)\right)^{-1}-U_{0}(\lambda)\right) \\
= & \left(\left(I-U(m, \lambda)+U_{0}(\lambda)\right)^{-1}-U_{0}(\lambda)\right)\left(\left(I-U(m, \lambda)+U_{0}(\lambda)\right)-U_{0}(\lambda)\right) \\
& \times\left(\left(I-U(m, \lambda)+U_{0}(\lambda)\right)^{-1}-U_{0}(\lambda)\right) \\
= & \left(I-U_{0}(\lambda)\left(I-U(m, \lambda)+U_{0}(\lambda)\right)-\left(I-U(m, \lambda)+U_{0}(\lambda)\right)^{-1} U_{0}(\lambda)+U_{0}(\lambda)^{2}\right) \\
& \times\left(\left(I-U(m, \lambda)+U_{0}(\lambda)\right)^{-1}-U_{0}(\lambda)\right) \\
= & \left(I-\left(I-U(m, \lambda)+U_{0}(\lambda)\right)^{-1} U_{0}(\lambda)\right)\left(\left(I-U(m, \lambda)+U_{0}(\lambda)\right)^{-1}-U_{0}(\lambda)\right) \\
= & \left(I-U(m, \lambda)+U_{0}(\lambda)\right)^{-1}-\left(I-U(m, \lambda)+U_{0}(\lambda)\right)^{-1} U_{0}(\lambda)\left(I-U(m, \lambda)+U_{0}(\lambda)\right)^{-1} \\
& -U_{0}(\lambda)+\left(I-U_{(}(m, \lambda)+U_{0}(\lambda)\right)^{-1} U_{0}(\lambda) \\
= & \left(I-U(m, \lambda)+U_{0}(\lambda)\right)^{-1}-U_{0}(\lambda)-U_{0}(\lambda)+U_{0}(\lambda) \\
= & \left(I-U(m, \lambda)+U_{0}(\lambda)\right)^{-1}-U_{0}(\lambda) .
\end{aligned}
$$

\section{Proof of Theorem 1.1}

According to general theory of linear equations solvability [3], we obtain that the problem (1.1), (1.2) is solvable for sets $\left\{h_{n}\right\}_{n} \in \mathbb{Z}_{+}$that satisfy the condition

$$
U_{0}(\lambda) g(m, \lambda)=0
$$

This condition along with Lemma 2.1 is equivalent to represantion (a) of the Theorem 1.1.

Under such a condition, all solutions of the problem (1.1), (1.2) have the form

$$
\begin{aligned}
x_{n}= & U(n, \lambda) U_{0}(\lambda) c+U(n, \lambda)(I-U(m, \lambda))^{-} g(m, \lambda)+g(n, \lambda) \\
= & U(n, \lambda) U_{0}(\lambda) c+U(n, \lambda) \sum_{k=0}^{\infty}(\mu-1)^{k}\left\{\sum_{l=0}^{\infty} \mu^{-l-1}\left(U(m, \lambda)-U_{0}(\lambda)\right)^{l}\right\}^{k+1} g(m, \lambda) \\
& -U(n, \lambda) U_{0}(\lambda) g(m, \lambda)+g(n, \lambda),
\end{aligned}
$$

which along with notations introduced is equivalent to representation (b) of the theorem. 


\section{Comments and Examples}

Remark 4.1. Suppose $B$ is Hilbert space, in such case we can show that formulas (2.10), (2.11) give us the representation for the Moore-Penrose pseudoinverse $[7,8]$ for $E-U(m, \lambda)$ with $U_{0}(\lambda)$ being self-adjoint operator (orthogonal projector) [6].

Remark 4.2. Supposing $A_{k}^{-1} \in \mathcal{L}(\mathbf{B}) \in L(B)$ exist for all $k=\overline{0, m-1}$, then the following equation holds: $\Phi(k, i, \lambda)=U(k, \lambda) U^{-1}(i, \lambda), k>i$. This allows representing the solutions of (1.1), (1.2) using only the family of operators $U(n, \lambda)$ and their inverse.

Let us illustrate the statements proved above on example of two-dimensional systems. (1) Consider equation

$$
\vec{x}_{n+1}=\lambda A_{n+1} \vec{x}_{n}+\vec{h}_{n+1}, \quad n \geqslant 0
$$

with periodicity condition

$$
\vec{x}_{3}=\vec{x}_{0}
$$

where $\vec{x}_{n}=\left(x_{n}^{1}, x_{n}^{2}\right)^{T}, x_{n}^{1}, x_{n}^{2} \in \mathbb{R}, \vec{h}_{n}=((3 \sqrt{3} r) / 4 \pi, 0)^{T}$,

$$
A_{n}=\left(\begin{array}{rr}
-\frac{1}{2} & -\frac{\sqrt{3}}{2} \\
\frac{\sqrt{3}}{2} & -\frac{1}{2}
\end{array}\right), \quad \forall n \geqslant 0
$$

It is easy to see that

$$
\vec{x}_{3}=\lambda^{3} \vec{x}_{0}+g(3, \lambda)
$$

where

$$
g(3, \lambda)=\left(\frac{-3 \sqrt{3} r \lambda-3 \sqrt{3} r \lambda^{2}+6 \sqrt{3} r}{8 \pi}, \frac{9 r \lambda-9 r \lambda^{2}}{8 \pi}\right)^{T} .
$$

Then the following hold for all $k \geqslant 0$

$$
U(3 k+1, \lambda)=\lambda^{3 k+1} A_{2}, \quad U(3 k+2, \lambda)=\lambda^{3 k+2}\left(\begin{array}{cc}
-\frac{1}{2} & \frac{\sqrt{3}}{2} \\
\frac{\sqrt{3}}{2} & -\frac{1}{2}
\end{array}\right), \quad U(3 k+3, \lambda)=\lambda^{3 k+3} E .
$$

By substituting periodicity condition (4.2) into (4.4) we obtain an equation depending on $\vec{x}_{0}$ :

$$
\left(1-\lambda^{3}\right) \vec{x}_{0}=g(3, \lambda)
$$




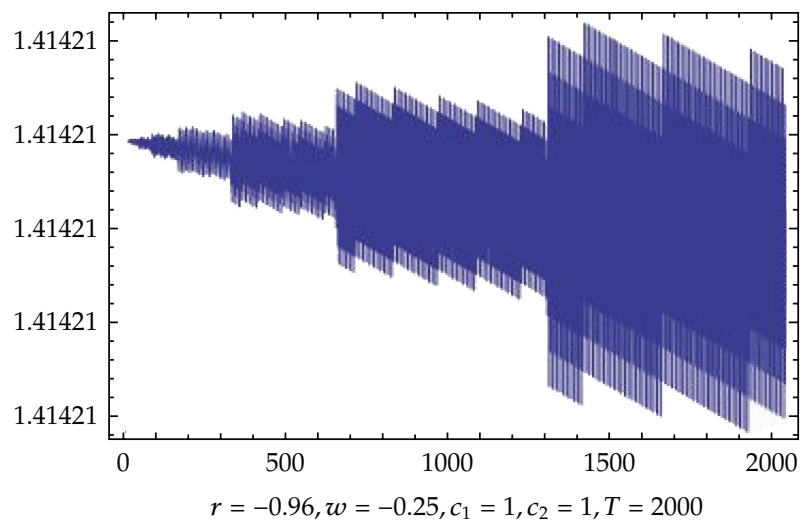

Figure 1

Consider the case when $\lambda=1$. In such case (4.7) turns into $\overrightarrow{0 x}_{0}=(0,0)^{T}$ which holds for arbitrary initial vector $\vec{x}_{0} \in \mathbb{R}^{2}$. Obviously $U^{n}(1,1)=U(n, 1)$ and $U_{0}(1)=E$. According to Theorem 1.1, all periodic solutions of (4.1) have the form

$$
\left(\begin{array}{l}
x_{n}^{1}\left(c_{1}, c_{2}\right) \\
x_{n}^{2}\left(c_{1}, c_{2}\right)
\end{array}\right)=\left(\begin{array}{cc}
\cos \frac{2 \pi}{3} n & \sin \frac{2 \pi}{3} n \\
-\sin \frac{2 \pi}{3} n & \cos \frac{2 \pi}{3} n
\end{array}\right)\left(\begin{array}{l}
c_{1} \\
c_{2}
\end{array}\right)+\left(\begin{array}{c}
\frac{3 r}{2 \pi} \sin \frac{2 \pi}{3} n \\
0
\end{array}\right),
$$

for all $\vec{c}=\left(c_{1}, c_{2}\right)^{T} \in \mathbb{R}^{2}$.

(2) We can search for periodic solutions of any period $w$ in previous problem. They have common view

$$
\vec{x}_{n}\left(c_{1}, c_{2}, w, r\right)=\left(\begin{array}{cc}
\cos \frac{2 \pi}{w} n & \sin \frac{2 \pi}{w} n \\
-\sin \frac{2 \pi}{w} n & \cos \frac{2 \pi}{w} n
\end{array}\right)\left(\begin{array}{l}
c_{1} \\
c_{2}
\end{array}\right)+\left(\begin{array}{c}
\frac{r w}{2 \pi} \sin \frac{2 \pi}{w} n \\
0
\end{array}\right)
$$

where $c_{1}, c_{2}, w, r$ are parameters.

To illustrate complexity of the set we did the following.

Recall that the length of vector $\vec{x}_{n}$ is $\ell \vec{x}_{n}=\sqrt{\left(\vec{x}_{n}^{1}\right)^{2}+\left(\vec{x}_{n}^{2}\right)^{2}}$. System (4.9) was implemented using the Wolfram Mathematica 7 framework. $x$-axis corresponds to time, while $y$-axis corresponds to the length of the vector. The length of the vector was calculated in the integer moments of time $n$. The points obtained in such way were connected in a piecewise linear way. The results obtained for particular values of the parameters are depicted on the following figures.

We can see how the trajectory of vector length densely fills rectangle or turns into a line (Figures 3 and 4). Figures 1, 2, 5, and 6 demonstrate that the trajectory can fill structured sets. The structure depicted on Figure 1 resembles fractal.

This allows us to conclude that behavior of the system is rather complex; it can undergo unpredictable changes with the slightest variations of a single parameter. We must admit that effects described need further theoretical investigation. 


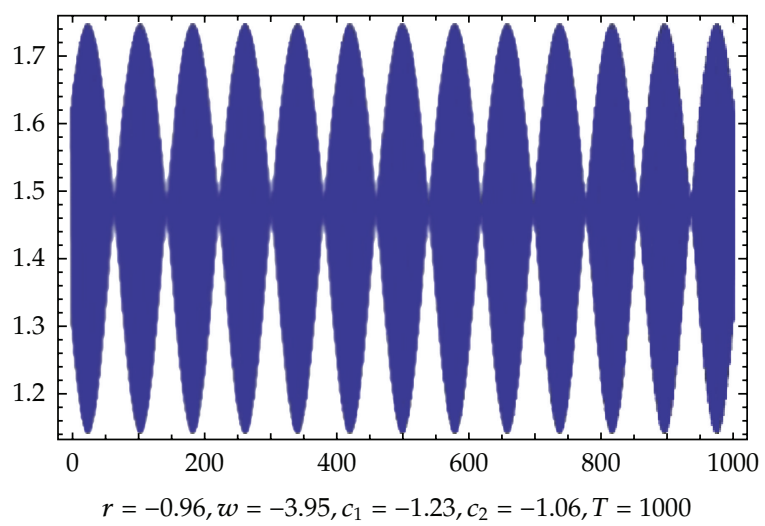

Figure 2

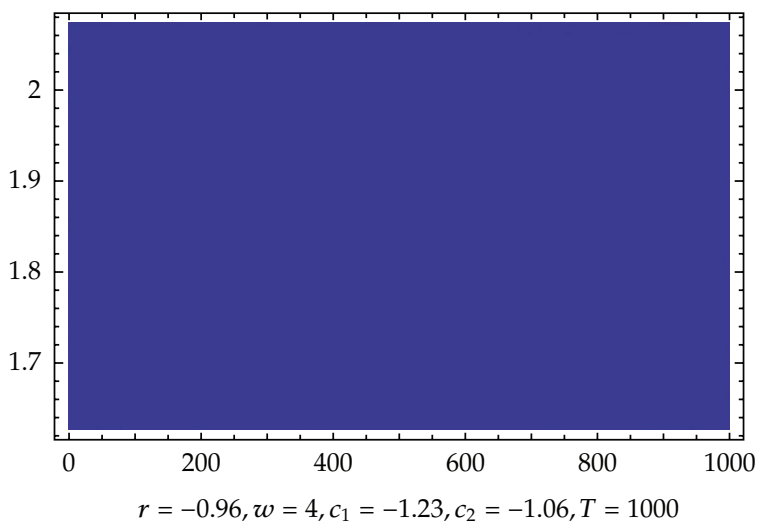

Figure 3

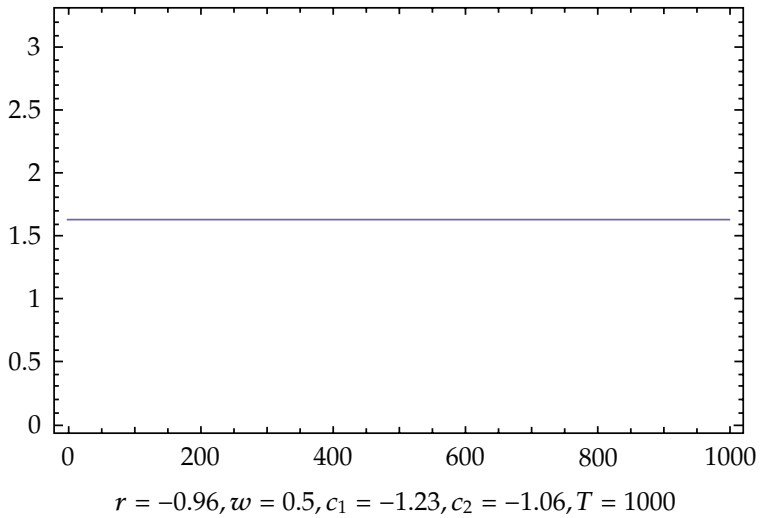

Figure 4 


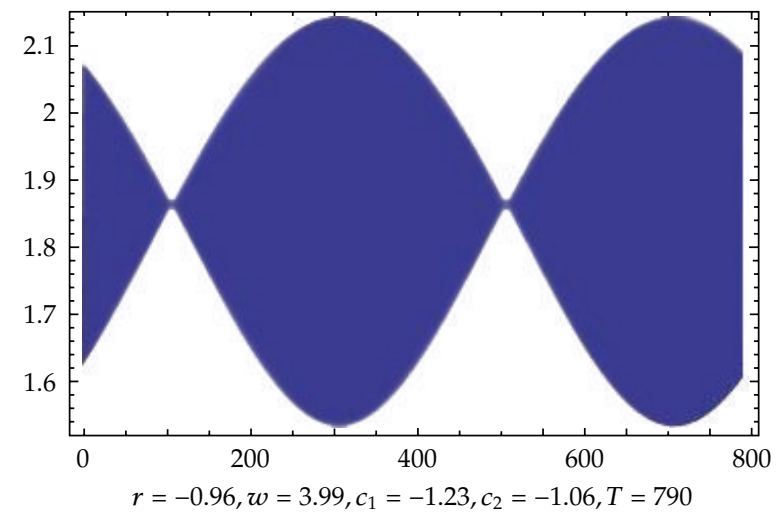

Figure 5

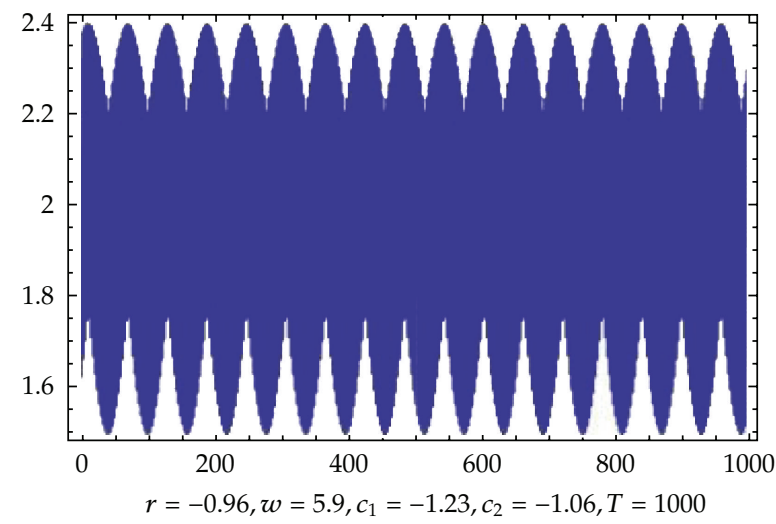

Figure 6

\section{Acknowledgment}

A. A. Boichuk was supported by the Grant 1/0090/09 of the Grant Agency of Slovak Republic (VEGA) and by the Project APVV-0700-07 of the Slovak Research and Development Agency.

\section{References}

[1] B. P. Demidovich, Lectures Mathematical Theory of Stability, Nauka, Moscow, Russia, 1967.

[2] Y. L. Daletskyi and M. G. Krein, Stability of Solutions of Differential Equations in Banach Space, Nauka, Moscow, Russia, 1970

[3] A. A. Boichuk and A. M. Samoilenko, Generalized Inverse Operators and Fredholm Boundary Value Problems, VSP, Utrecht, The Netherlands, 2004.

[4] I. D. Chueshov, Introduction to the Theory of Infinite-Dimensional Dissipative Systems, ACTA, Kharkov, Ukraine, 2002.

[5] A. A. Boichuk and A. A. Pokutnyi, "Criterion of solvability of difference equations in Banach space," in Bulgarian-Turkish-Ukrainian Scientific Conference Mathematical Analysis, Differential Equations and Their Applications, pp. 241-247, Prof. Marin Drinov Academic Publishing House, Sunny Beach, Bulgaria, September 2010. 
[6] K. Iosida, Functional Analysis, Springer, Berlin, Germany, 1965.

[7] E. H. Moore, "On the reciprocal of the general algebraic matrix (Abstract)," Bulletin of the American Mathematical Society, no. 26, pp. 394-395, 1920.

[8] R. Penrose, "A generalized inverse for matrices," Mathematical Proceedings of the Cambridge Philosophical Society, vol. 51, no. 3, pp. 406-413, 1955. 


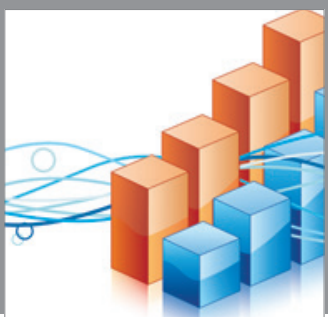

Advances in

Operations Research

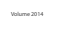

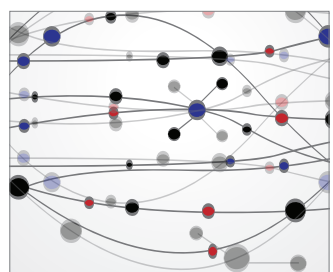

\section{The Scientific} World Journal
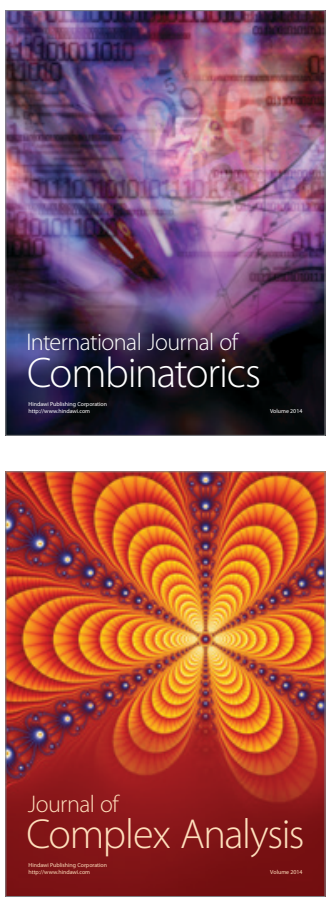

International Journal of

Mathematics and

Mathematical

Sciences
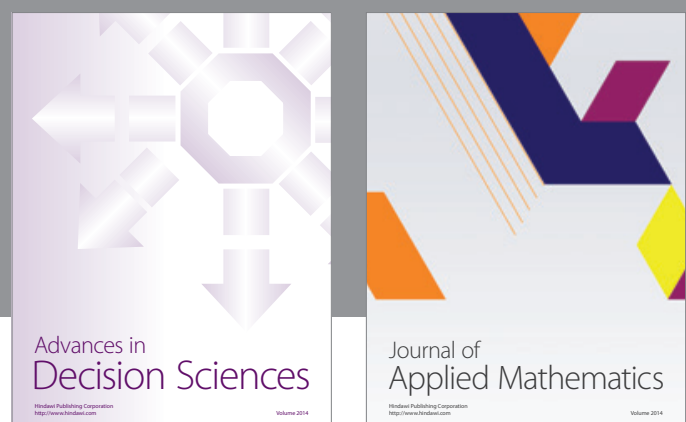

Journal of

Applied Mathematics
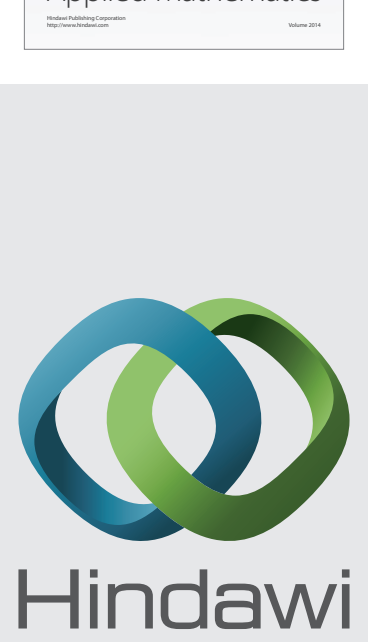

Submit your manuscripts at http://www.hindawi.com
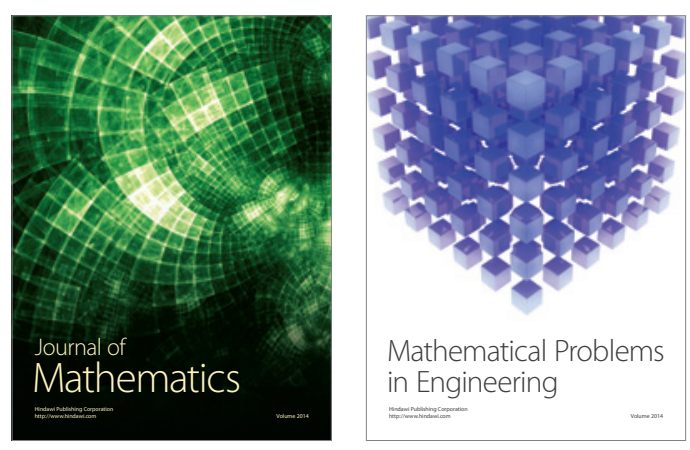

Mathematical Problems in Engineering
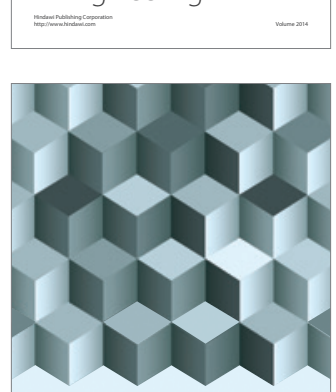

Journal of

Function Spaces
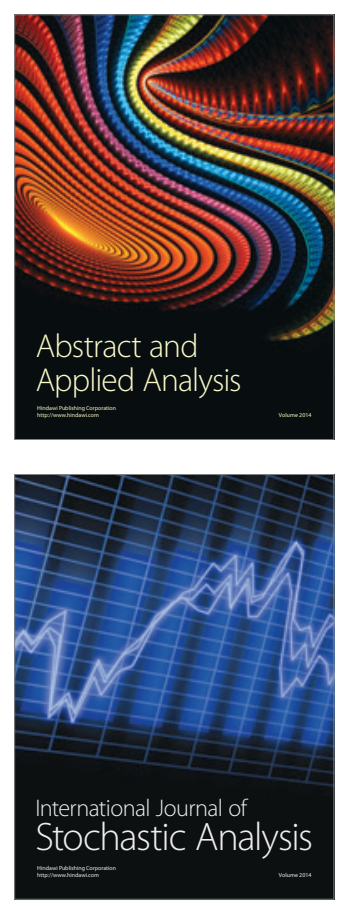

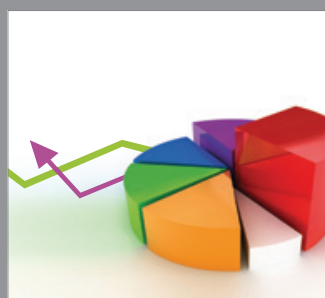

ournal of

Probability and Statistics

Promensencen
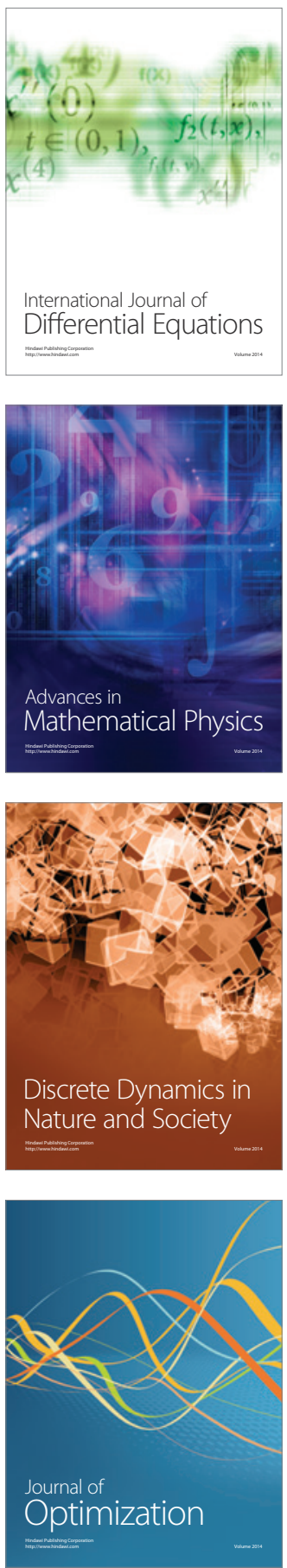